\title{
Linx
}

Revue des linguistes de l'université Paris X Nanterre

$76 \mid 2018$

Dire l'humain : les noms généraux dénotant les

humains

\section{Le nom d'homme est-il un nom général ?}

\section{Catherine Schnedecker}

\section{(2) OpenEdition}

Journals

Édition électronique

URL : http://journals.openedition.org/linx/2506

DOI : $10.4000 /$ linx.2506

ISSN : 2118-9692

Éditeur

Presses universitaires de Paris Nanterre

\section{Édition imprimée}

Date de publication : 31 juillet 2018

Pagination : 23-56

ISSN : 0246-8743

\section{Référence électronique}

Catherine Schnedecker, «Le nom d'homme est-il un nom général ? », Linx [En ligne], 76 | 2018, mis en ligne le 31 janvier 2019, consulté le 19 avril 2019. URL : http://journals.openedition.org/linx/2506 ; DOI : 10.4000/linx.2506

Ce document a été généré automatiquement le 19 avril 2019

Département de Sciences du langage, Université Paris Ouest 


\title{
Le nom d'homme est-il un nom général ?
}

\author{
Catherine Schnedecker
}

\section{Introduction}

1 Cette étude fait suite à une série de travaux (Cappeau et Schnedecker, 2014a, b ; 2015, 2016; Mihatsch, 2015a, 2015b) consacrés à une sous-catégorie de noms, encore en marge des études dans le domaine de la sémantique nominale et qui ont pour particularité de dénommer des humains (footballeur, universitaire, terroriste, président, champion, etc.). Parmi ceux-ci figure une liste de noms (homme, personne, individu, gens, ....) qu'on qualifie de noms généraux, en vertu d'un sens qu'on peut dire "général ». Ils présentent en effet deux caractéristiques sémantiques principales. Premièrement, ils sont considérés (dans les dictionnaires ou la littérature sur la question, cf. infra) comme synonymes du nom homme, lui-même doté, dans les analyses componentielles «classiques ", de traits sémantiques en nombre restreint : [+humain], [+mâle], [+adulte], d'où le sens " général ». Deuxièmement, ils sont/peuvent, à ce titre, être utilisés comme $\mathrm{N}$ dits hyperonymes/classifieurs/ descripteurs, notamment dans les définitions dites par le genre prochain d'autres noms d'humains (cf. 1-3) :

(1) Soldat: Homme qui sert dans une armée à quelque titre que ce soit (TLFi)

(2) Roi : Homme qui règne (politiquement). (TLFi)

(3) Mécanicien : Homme chargé de la conduite et de l'entretien des machines et des moteurs à bord (TLFi)

2 Dans le cadre du projet NHUMA ${ }^{1}$ consacré à l'étude des noms d'humains (désormais $\mathrm{NH}$ ) dont participe ce travail, nous avons pris l'habitude de diviser ces $\mathrm{NH}$ en deux grandes catégories principales: les N que nous appelons « généraux » et les autres, qui sont, par contraste, spécifiques. Or, on peut se demander - et c'est la première question qui nous intéressera ici - ce qui motive cette terminologie, sachant que, d'une part, on vient de le voir, il existe dans la littérature tout un ensemble de termes qui paraissent, au niveau métalinguistique, synonymes de "général " tels que hyperonyme, classifieur, descripteur, etc. et que, d'autre part, il existe aussi tout un ensemble de travaux dédiés à la question 
des noms généraux. La question est donc de savoir comment les études dédiées aux noms généraux caractérisent ceux-ci, s'il y est question des $\mathrm{NH}$ et quelle est la place qui leur y est, le cas échéant, dévolue.

En second lieu, on peut se demander quels sont les noms qui constituent la classe des $\mathrm{NH}$ généraux. En effet, force est de constater que leur liste varie selon les auteurs et les dictionnaires. C'est ainsi que Gross $(1995,2008)$ et Le Pesant $(2002)$ considèrent comme « classifieurs » des NH tels que :

(4) Type, mec, être, gens, gars, gus, homme

au vu d'une triple propriété : i) l'incapacité à servir d'attribut (5), ii) la capacité à servir d'anaphore à des $\mathrm{NH}$ (6), et iii) la possibilité de se voir remplacer par certains pronoms indéfinis (7-8) :

(5) *Paul est un type/ Les pauvres sont des gens (ex. de Gross, 2008)

(6) Le facteur venait de passer. Ce (type, gars, mec, gus) fait sa tournée avec une régularité remarquable (ex. de Gross, 2008) ${ }^{2}$

(7) Alors surgit (un homme/quelqu'un) qui m'a salué (ex. de Gross, 2008)

(8) (Des gens sont venus/on est venu) me chercher. (ex. de Gross, 2008)

Quant aux dictionnaires, leur inventaire est tout aussi variable ${ }^{3}$ :

(9) Anthropoïde, hominoïde, individu, personne, quidam, humanité, gens, vulgaire, monsieur, bonhomme, gars, keum, mec, type, créature, mortel, âme, esprit, bonhomme, jules, mec (Petit Robert, 2016)

(10) Individu humain, mari, concubin, amant, fils, ... (TLFi)

Doit-on considérer l'ensemble des termes qui viennent d'être cités comme relevant d'une même catégorie de noms? Rien n'est moins sûr : des noms comme gars, mec et gus sont ressentis - et stigmatisés par les dictionnaires - comme émanant d'un registre de langue peu formel et, à ce titre, ils sont sans doute soumis à des conditions d'emplois plus contraintes qu'il n'y paraît $t^{4}$. Pour adresser au receveur des postes une réclamation à propos du facteur, on dirait, semble-t-il (12) de préférence à (11) :

(11) Le facteur vient de passer. Ce (type, gars, mec, gus/cette créature/Cet anthropoïde) a oublié de relever mon courrier

(12) Le facteur vient de passer. Cet homme/cette personne a oublié de relever mon courrier

Bref, comme on le voit la constitution d'une liste de NH généraux n'est pas acquise.

Partant, nous procéderons en deux temps. Nous commencerons par rappeler les caractéristiques des noms généraux, telles que mises au jour par les travaux pionniers de Halliday \& Hasan (1976), suivis, entre autres, par Schmid (2000:10-20) et Mahlberg (2005 : 5-11). Dans un second temps, nous situerons les NH au sein de cette catégorie. Pour ce faire, nous nous appuierons sur une étude de corpus visant à dégager les emplois les plus usuels d'homme et surtout à vérifier si son comportement se conforme ou non aux noms généraux.

\section{Les noms généraux : un état de la question}

Les noms dits généraux ont fait l'objet sinon de nombreuses études (cf. Schmid, 2000 ; Mahlberg, 2005, notamment) du moins de nombreuses observations (Lyons, Haliday \& Hasan, entre autres), dont nous synthétisons ici le propos en faisant ressortir cinq principales caractéristiques. 


\subsection{Caractéristiques sémantiques}

10 La première caractéristique tient à leur valeur sémantique même, qui est dite " générale », « insaisissable » (cf. les citations ci-dessous) « non spécifiée » (Schmid, 2000 : 6) ou encore « vague » (Partington, $1988: 91)$ :

(...) the elusiveness of meaning makes "the fact that general nouns are very general in meaning" (Halliday \& Hasan, 1976: 276) very difficult to grasp. (Mahlberg, 2005 : 1) Semantic generality or unspecificity (Schmid, $2000: 6$ )

11 C'est ce que reflètent les étiquettes qui leur sont accolées : low content words (Bolinger, 1977 : 5-6), shell nouns (Schmid, op. cit. : 6), unspecific nouns (Winter, 1992). Par le fait, ils sont fréquemment assimilés à des noms superordonnés ou hyperonymiques comme le montre le propos de Halliday \& Hasan (1976) ci-dessous :

On trouve par contre un ensemble de léxèmes très généraux - 'personne', 'individu', 'animal', 'bête', 'insecte', 'chose', 'truc', 'machin', 'endroit', 'matière', 'qualité', etc.- qui sont superordonnés à des sous-ensembles plus ou moins grands de ces (= tous les membres de n'importe laquelle des sous-classes qu'on connait habituellement en français) sous-classes de noms. (Lyons, $1978: 242$ )

From a lexical point of view, they are the superordinate members of major lexical sets (Haliday \& Hasan, 1976: 275)

12 Les exemples "paradigmatiques » de noms généraux proposés par Halliday \& Hasan, et abondamment repris par les études ultérieures, sont donnés en (13). On notera que les noms d'humains figurent en tête de liste :

(13) People, person, man, woman, child, boy, girl [human]

Creature [non-human animate]

Thing, object [inanimate concrete noun]

Stuff [inanimate concrete mass]

Business, affair, matter [inanimate abstract]

Move [action]

Place [place]

Question, idea [fact]

De là découlent quatre autres caractéristiques.

\subsection{Des noms « borderline » entre noms et pronoms}

Premièrement, la pauvreté des informations sémantiques de ces noms et, partant, leur extension, due au fait qu'ils sont assimilés également à des hyperonymes, se trouve aussi dévolue généralement aux pronoms. C'est ce qui explique que les noms généraux sont situés à la frontière entre unités lexicales et grammaticales. Qui plus est, ils sont censés commuter avec les pronoms, même si cette possibilité est plus limitée dans les faits qu'elle ne l'est en théorie, selon Mahlberg $(2005: 10)$ et Halliday \& Hasan :

A general noun is itself a borderline case between a lexical item (member of an open set) and a grammatical item (member of a closed system) (Halliday \& Hasan, 1976: 274)

I turned to the ascent of the peak. [The ascent/the climb/the task/the thing/it] is perfectly easy. (ex. de Halliday \& Hasan, op. cit.: 279)

On the borderline between grammatical and lexical cohesion is the cohesive function of the class of GENERAL NOUN. We can speak about a borderline here because a general noun is itself a borderline case between a lexical item (member of 
an open set) and a grammatical item (member of a closed system). (Halliday \& Hasan, 1976: 274)

(...) a sizable vocabulary of nouns more or less stereotyped as classifiers (...), sharing coreferential functions with pronouns" (Bolinger, 1977: 50)

Cette position est explicitée très justement par Schmid comme suit :

Full-content nouns have an enormous potential of detailed characterization of what speakers want to talk about, (...) have a more or less stable and rich denotation. (...) [They] are the main means of describing persons and objects, animals and plants, activities and events, and properties and circumstances. Pronouns with anaphoric function (...) have a very limited potential for characterization, if any at all. (...) They characterize their referents only with respect to a very small number of semantic dimensions: speaker vs. addressee vs. other roles, human vs. non-human, singular vs. plural, and male vs female. (...) Shell nouns hold a middle position between these extremes. To a certain extent, speakers can indeed use them to characterize a piece of experience, say as a fact, a problem, an idea or an aim. Like full-content nouns, shell nouns derive their potential for characterization from their denotation. (Schmid, $2000: 15$ )

\subsection{Des noms à fort potentiel cohésif}

La seconde caractéristique tient à ce que ces noms, comme les pronoms auxquels ils sont assimilés, ont un potentiel cohésif assez fort du fait qu'ils peuvent être utilisés comme anaphore (Halliday \& Hasan, op. cit.: 275) (cf. l'exemple 12 supra) ou cataphore (Partington, cité par Mahlberg, op. cit. :10) :

There are also things it is more difficult for parents to get a grip on, such as empathy, and the ability to retain ideas and hold them despite everybody else telling them (Partington, 1998: 92 in Mahlberg, 2000: 10)

Francis (1986) parle d'ailleurs à cet égard d'anaphoric nouns (A-nouns) et Schmid de «fonction de liage/liant » (linking ${ }^{5}, 2000: 15$ et pp. (pppp18-19).

Cet emploi cohésif se caractérise, formellement, par différentes marques linguistiques, dans la construction de leur syntagme d'accueil: présence des déterminants défini, démonstratif (Halliday \& Hasan, op. cit.) ou indéfini ( $a$, such, Partington in Malhlberg, op. cit., 9) et celle de modifieurs à sens modal (« only adjectives with an attitudinal meaning can occur » Hallyday \& Hasan, op. cit) ${ }^{6}$ :

(13) I've been to see my great-aunt. The poor old girl's getting very forgetful these days (ex. de Halliday \& Hasan, 1976: 276, notre numérotation)

Enfin la portée de ces $\mathrm{N}$ en emploi anaphorique peut être locale ou plus large, comme le relève Mahlberg (op. cit. : 8). De ce fait, ils peuvent aussi jouer le rôle d'anaphores dites "résomptives ", i.e. d'anaphores visant à reprendre et résumer à la fois le propos antérieur :

The interesting point about general nouns seems to be the way in which they refer back not only to previous nouns phrases but also to longer stretches of texts (Mahlberg, op. cit.: 8)

\subsection{Des $\mathrm{N}$ dont le sémantisme est dépendant du contexte d'occurrence}

De là vient que le sémantisme de ces $\mathrm{N}$ peut être fortement dépendant du contexte ${ }^{7}$, comme le soulignent Mahlberg (op. cit. : 8 ) et Schmid (2000: 13) : 
Their meaning depends on other elements in the context. According to Francis (1994:88), for instance, a noun that can function as a label is 'unspecific and requires lexical realization in its immediate context'. (Mahlberg, 2005: 8)

Their interpretation crucially depends on the shell content which must be expressed in the context, or at least be inferrable from it. (Schmid, 2000: 18)

(...) whether a given noun is a shell noun or not does not depend on inalienable

characterisitcs inherent in the noun, but on its use. (Schmid, $2000: 13$ )

Un exemple emprunté à Francis (cité par Mahlberg, ibid.) servira d'illustration :

(14)

(...) the patient's immune system recognized the mouse antibodies and rejected him. This meant they did not remain in the system long enough to be fully effective.

The second generation antibody now under development is an attempt to get around this problem by 'humanising' the mouse antibodies, using a technique developed by... (ex. de Francis in Mahlberg, 2005 : 9)

Schmid précise d'ailleurs, à cet égard, qu'il n'y aurait pas tant de noms généraux (shell nouns dans sa terminologie) en soi que des noms qui sont fréquemment utilisés comme tels. De sorte qu'il est impossible d'en fournir la liste exhaustive.

Dans ce genre d'emploi, ces noms fonctionnent, ainsi que l'observe Mahlberg (op. cit.), comme des signaux textuels balisant des unités d'informations, comme des étiquettes permettant d'évaluer des portions discursives, comme des termes métalinguistiques, etc.

\subsection{Des $\mathrm{N}$ comportant une dimension interpersonnelle}

Une dernière particularité des noms généraux, signalée initialement par Halliday \& Hasan, tient à ce qu'ils sont susceptibles de véhiculer « an interpersonal element into the meaning » (op. cit. : 276), « a particular attitude of the part of the speaker». Tel que défini par les auteurs, cet élément interpersonnel consiste en ceci :

Essentially the attitude conveyed is one of familiarity, as opposed to distance, in which the speaker assumes the right to represent the thing is referring, to as it impinges on him personally; hence the specific attitude may be either contemptuous or sympathetic, the two being closely related as forms of personal involvement. (Halliday \& Hasan, op. cit.: 276)

They are frequently used in spoken language and "enable a speaker to express attitudes and feelings without needing to locate an exact or precise referent" (Carter \& Mc Carthy 1997, 16 in (Mahlberg, 2005, 9).

Dans une perspective similaire, Schmid (op. cit. : 14) évoque une fonction sémantique des «shell nouns" consistant à «characterizing and perspectivizing complex chunks of informations" .

Pour terminer ce survol rapide, le schéma proposé par Schmid qui situe les noms généraux à la croisée des noms et des pronoms, synthétise à la fois leurs dimensions sémantiques et fonctionnelles. 


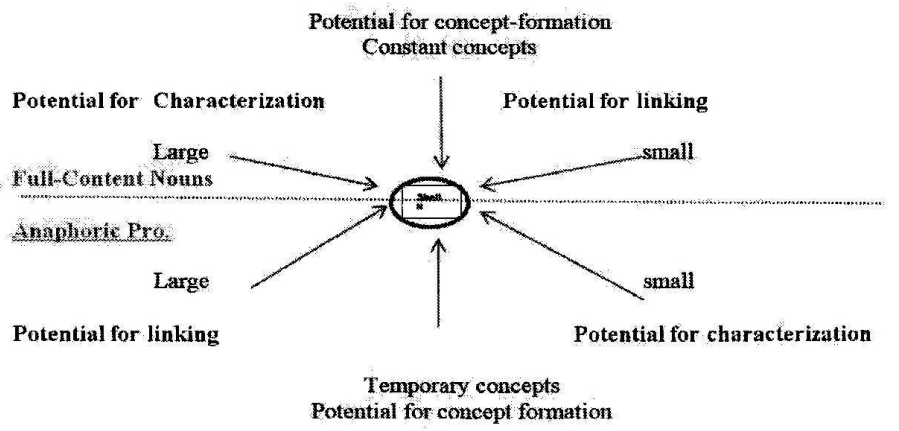

FIgURE 1. Schéma de H. J. Schmid représentant la place et les fonctions des NGH entre Noms et Pronoms (2000: 19)

\section{La place des noms d'humains généraux dans les NG}

Compte tenu de la variété des noms considérés comme généraux, il n'est guère étonnant que tous ne partagent pas unanimement les propriétés qui viennent d'être rappelées.

Il suffit, en effet, de considérer la liste princeps établie par Halliday et Hasan (rappelée cidessus) pour se rendre compte que, du point de vue des principes classificatoires désormais standards pour les noms (cf. Flaux et Van de Velde, 2000, entre autres), les noms généraux ne constituent pas une classe homogène ${ }^{8}$ : certains sont comptables ( people, person, man, woman, child, boy, girl, creature, thing, object), d'autres massifs (stuff); certains sont concrets (people, person, man, woman, child, boy, girl, creature, thing, object, stuff, place), d'autres abstraits (business, affair, matter, move, question, idea), ce qui augure de comportements différents.

Force est de constater, par ailleurs, que, dans les développements récents (Legallois, 2008; Flowerdew, 2002) et au vu des dénominations qui leur sont accolées (shell nouns, signalling nouns, noms sous-spécifiés, carrier nouns, métalanguage nouns), l'intérêt se concentre sur des N plutôt abstraits, à fonction méta-(discursive ou langagière) (problème, raison, idée, ...) ce qui tendrait à marginaliser les $\mathrm{NH}$.

En effet, ceux-ci sont dotés d'un contenu descriptif relativement stable, plus en tout cas que des $\mathrm{N}$ comme fait, idée ou problème. Cela se traduit notamment par le fait qu'ils n'ont pas les mêmes constructions syntaxiques que les "shell nouns" de Schmid, ce que l'auteur observe lui-même p. 3 :

(15) *The boy that I had no money (exemple de Schmid, op. cit.: 3)

(16) *The boy was that I had no money (exemple de Schmid, op. cit.: 3)

(17) The fact that I had no money; The fact was that I had no money (exemple de Schmid, op. cit.: 3 ) 
31 En effet, le sémantisme vague ou sous-spécifié (cf. Legallois, 2008) d'un nom comme fact appelle une forme de complémentation propositionnelle ${ }^{9}$, qui vient en quelque sorte saturer la référence, ce qu'exclut un N comme boy dans les exemples (16-17, supra).

Bref, au regard des diverses sous-catégories de noms généraux établies et de l'évolution des études les concernant, la place pour des $\mathrm{NH}$ du type de homme, femme, etc. paraît de plus en plus marginale, même s'ils partagent avec les noms généraux un certain nombre des points communs énumérés ci-après. Premièrement, les noms généraux sont souvent assimilés, fonctionnellement et sémantiquement, à des pronoms, par certains auteurs :

A la limite peuvent aussi figurer dans cette catégorie (= celle des hyperonymes ou noms génériques) les anaphoriques pronominaux il(s) et elle(s) : marqués en genre, ceux-ci équivalent en effet pragmatiquement à des hyperonymes, recouvrant l'un toute la classe des noms masculins et l'autre toute celle des noms féminins (M.-J [Reichler-] Béguelin, 1989 : 306)

Il me semble ainsi que le plus souvent, l'anaphore se ramène pour l'essentiel à un simple passage à l'hyperonyme (c'est en cela qu'on peut y voir, par rapport à la répétition intégrale, un procédé d'économie) (...) (A. Berrendonner, $1983: 236$ )

Deuxièmement, ils servent d'anaphore, ce qu'illustrent les extraits ci-dessous :

(18) Samedi, vers $19 \mathrm{~h}$, la victime âgée de 29 ans et connue pour sa fragilité, avait pris son vélo pour se rendre sur un terrain vague de Blainville. L'homme s'était aspergé d'essence avant d'y mettre le feu. Malgré ses brûlures, il avait réussi à remonter sur sa bicyclette pour rejoindre une route proche. Là, un automobiliste s'est arrêté. Ce témoin a aussitôt prévenu les secours et la gendarmerie. (Républicain Lorrain, 20/07/2011, in Schnedecker, 2015)

(19) Arrivé au milieu de ses élèves, Erwan Fauré, regard gris azur, signale sa présence d'un "bonjour" lâché d'une voix douce mais qui porte. Les enfants s'interrompent, sentant le regard du professeur posé sur eux. On voit l'homme heureux d'être là. Les enfants aussi. Ils se tiennent prêts, main gauche sur le manche, main droite fermée sur l'archet. Silence. (Le Monde, 26/06/03, in Schnedecker 2005, 2015)

Enfin, ils peuvent traduire une forme de point de vue ou d'attitude de proximité vs de distance de la part du locuteur par rapport au référent visé. Comme nous l'avons montré (Schnedecker, 2015), les anaphores à nom humain général sont sujettes à un certain nombre de contraintes d'ordre pragmatique. Elles prévalent, semble-t-il, dans des contextes où est introduit un référent sur lequel le locuteur n'a donné aucune information en dehors de son "genre" (homme vs femme, p.ex.), et ce, jusqu'à l'identification du référent et, en particulier, sa désignation par un nom propre, comme dans (22a) :

(20a) La police de Grampian, qui patrouillait la portion de l'A39 bloquée par la neige entre Braemar et Spittal of Glenshee, jeudi soir, a repéré une voiture apparemment abandonnée sur le bas-côté, au pied de la station de ski de Glenshee. En s'approchant, les policiers ont découvert le chauffeur inconscient dans son véhicule. (...).

L'homme, qui souffrait d'une hypothermie grave, a dû être transporté par hélicoptère à la Royal Infirmary d'Aberdeen. Il a pu être identifié un peu plus tard comme Mr. Maxwell Sim, âgé de 48 ans et domicilié à Watford, en Angleterre. Mr Sim était un VRP employé en freelance par la société Guest, de Readin, spécialiste de produits d'hygiène bucco-dentaire écologiques. (...).

Désormais tout à fait rétabli, Mr. Sim serait rentré chez lui à Watford. (J. Coe, La vie très privée de Mr Sim, incipit) (exemple 32 in Schnedecker, 2015 : 52-53)

Cela étant, une fois le référent identifié, le retour à l'hyperonyme (20b) ne semble plus guère adapté à la situation et peut paraître moins naturel que (20a) : 
(20b) Mr Sim était un VRP employé en freelance par la société Guest, de Readin, spécialiste de produits d'hygiène bucco-dentaire écologiques. 濫...嚂. Désormais tout à fait rétabli, l'homme serait rentré chez lui à Watford.

\section{(21):}

(21) (p1) Pierre est venu. (p2) Cet homme est sympathique. (Gross, $2008: 29)$

En effet, l'emploi du nom propre par le locuteur laisse entendre une forme de familiarité qu'il entretient avec le dénommé Pierre (cf. Ariel, 1990) et donc qu'il dispose sur celui-ci d'un certain nombre de connaissances. Or, le recours à un nom d'humain général en guise de reprise anaphorique paraît, pragmatiquement, d'autant plus incongru que :

1. il marque une forme zéro de la connaissance contraire à ce qui ressort de l'environnement textuel ;

2. le prédicat de la (p2), qui dénote un jugement de valeur fondé sur une relative connaissance du référent, ne justifie pas non plus l'anaphore à noms général humain ;

3. il introduit une sorte de distance entre le référent et le locuteur.

Dès l'instant où cette distance est « légitimée » par le contexte comme, semble-t-il, en (22), l'anaphore à tête lexicale de NH général passe mieux selon nous :

(22) (p1) Pierre m'a déçue terriblement aux dernières présidentielles. (p2) Cet homme vote quoi qu'il arrive pour le FN

Bref, au terme de cette partie, et sur les trois critères examinés, les NH semblent présenter des points communs avec les noms qualifiés communément de généraux, modulo la question, qui n'est pas triviale, de l'extension de la catégorie et de leurs souscatégories.

\section{Etude de cas : le nom d'homme}

Comme on s'en souvient la liste princeps de noms généraux proposée par Halliday \& Hasan comprend, en première ligne, les NH people, person, man, woman, child, boy, girl. En prenant comme expression-témoin le $\mathrm{NH}$ d'homme, nous allons vérifier dans quelle mesure il satisfait aux propriétés des noms généraux qui viennent d'être évoquées. Dans ce but, nous avons constitué un corpus de 400 occurrences du nom homme, subdivisées en 200 occurrences au singulier et 200 au pluriel. Ces occurrences sont tirées de la base de données Wortschat ${ }^{10}$, qui représente un usage de la langue proche du français contemporain standard, beaucoup moins formel que Frantext. Nous procédons à l'analyse du corpus selon les niveaux morphologique, syntaxique et sémantique.

\subsection{Niveau morphologique}

41 Le premier point à souligner, et il aura son importance, est que le masculin en tant qu'expression du trait [+ mâle] est prédominant dans l'ensemble des emplois : totalement au singulier et à hauteur de $85 \%$ pour les emplois au pluriel. On ne dénombre, en effet, que 32 cas où ce trait est neutralisé et où homme dénote l'espèce humaine. Il s'agit alors de contextes à caractère religieux ou encyclopédico-historique (23-25) :

(23) Dès la plus lointaine Antiquité, des hommes venaient ici pour prier, adorer, servir Dieu et leurs frères. (www.saint-loup.ch, 0002-11-30)

(24) L'hygiène de vie d'inspiration biblique et scientifique est importante pour mieux servir Dieu et les hommes. (renens.adventiste.ch, 0002-11-30) 
(25) Les hommes du néolithique savaient choisir leurs lieux de villégiature. (ennedi.free.fr.txt, 2007-10-22)

\subsection{Niveau syntagmatique}

42 Au niveau de la distribution interne au SN, un premier clivage se fait ressentir entre les emplois au singulier vs pluriel d'homme.

\subsubsection{Modification et types de modifieurs vs absence de modifieurs}

La différence la plus marquante entre le singulier et le pluriel tient à la répartition entre emplois non modifiés vs modifiés (cf. Tableau 1):

\begin{tabular}{|l|l|l|l|l|l|}
\hline & Sans modif. & \multicolumn{3}{|l|}{ Avec modifieur } & Sous total \\
\hline & & adjectif & Ct du nom & relative & \\
\hline homme & $20 \%$ & $54 \%$ & $20 \%$ & $6 \%$ & $80 \%$ \\
\hline hommes & $50 \%$ & $22 \%$ & $26 \%$ & $2 \%$ & $50 \%$ \\
\hline
\end{tabular}

Tableau 1. Répartition des modifieurs d'homme au sg/pl.

$$
\begin{aligned}
& \text { modifieurs se répartissent de manière équilibrée entre adjectifs et compléments du nom } \\
& \text { et } 10 \% \text { d'entre eux sont constitués par des formes lexicalisées du type hommes politiques, } \\
& \text { hommes mariés, hommes d'affaire, hommes de main, ... }
\end{aligned}
$$

(26) Mes hommes de main posent des égouts, posent de l'asphalte et construisent des maisons. (matin.branchez-vous.com, 2009-09-13)

(27) On aurait beau ordonner des hommes mariés, cela ne résoudrait pas les problèmes de l'Église catholique. (www.cyberpresse.ca, 2006-02-28)

Au singulier, les SN sont modifiés dans $80 \%$ des cas, avec un pourcentage de séquences polylexicales trois fois plus élevé qu'au pluriel. On en dénombre 35\%. Parmi les plus fréquentes, $18 \%$ sont constituées par jeune homme et $5 \%$ par homme d'affaire. On note aussi $2 \%$ d'homme fort, et $2 \%$ d'homme politique.

Par ailleurs, pour le singulier, 15,5\% des adjectifs sont descriptifs. Ils fournissent alors un certain nombre de caractéristiques physiques ou morales du référent(28). 2,5\% d'entre eux sont des participes passés dénotant des actions répréhensibles (manifestement tirées de faits divers) (cf. 29-30). Enfin, le nombre de modifieurs des SN au singulier peut aller de 2 (30\% des occ.) à 3 (8\%) alors que celui des SN pluriels n'excède pas 2 (6\%) :

(28) Boulanger est un homme calme et rangé, d'allure propre, sans aucun tatouage et en bonne forme physique. (www2.canoe.com, 2009-04-17)

(29) Arrivée sur les lieux, la police remarque un homme dissimulé derrière des bosquets et interpelle l'individu. (www.tdg.ch, 2011-10-20)

(30) Un homme soupçonné d'avoir tué la journaliste Anna Politkovskaïa s'est fait délivrer un faux passeport, par une femme travaillant au Service fédéral des Migrations en Tchétchénie, en vue de quitter la Russie. (rss.feedsportal.com, 2009-03-12) 


\subsubsection{La détermination}

47 Les modalités de la détermination contribuent encore à nuancer le paysage entre les formes au singulier et au pluriel et, partant, se révèlent beaucoup plus intéressantes.

Il ressort du tableau (2) ci-dessous que, globalement, la distribution des déterminants opère de façon presque complémentaire. Les $\mathrm{SN}$ du singulier se réalisent au moyen de déterminants 0 (dans des proportions qui vont du simple au double : $13 \%$ au singulier contre 7 au pluriel), l'indéfini ( $49,5 \%$ au sg contre 9,5\% au pluriel, donc cinq fois moins) ou le démonstratif, peu - ou quasiment pas -, représenté au pluriel (1\% contre $13 \%$ au singulier).

Inversement, les $\mathrm{SN}$ au pluriel sont constitués principalement de déterminants quantifieurs dans $20 \%$ des cas (absents au singulier) ou de définis (dans des proportions qui vont du simple au double par rapport au singulier).

50 Nous allons examiner en détail ces chiffres en commençant par les cas de quasi égalité pour finir par les emplois les plus contrastés.

\begin{tabular}{|l|l|l|l|l|l|l|}
\hline & 0 & IND & QUANT & DEF & DEM & POSS $^{11}$ \\
\hline homme & $\mathbf{1 3 \%}$ & $\mathbf{5 0 \%}$ & $0 \%$ & $20 \%$ & $\mathbf{1 3} \%$ & $3 \%$ \\
\hline hommes & $7 \%$ & $9,5 \%$ & $\mathbf{2 7} \%$ & $53,5 \%$ & $1 \%$ & $2 \%$ \\
\hline
\end{tabular}

Tableau 2. Répartition des déterminants d'homme au sg/pl.

\subsubsection{Dét possessif + homme : diversité des interprétations}

51 Le déterminant possessif arrive pratiquement à égalité dans les $\mathrm{SN}$ au singulier et au pluriel. Cela étant, cette répartition équilibrée ne doit pas occulter des différences qualitatives réelles.

52 C'est ainsi que le possessif au pluriel renvoie à l'idée d'une appartenance communautaire (équipe, armée, corps constitué) (31-32) :

(31) Notre défense militaire est ridiculisée, lorsque nos hommes sont bloqués a une frontière ou que notre seul et unique porte-avions reste à quai.. (a19.free.fr.txt, 2007-10-22)

(32) Quand elles sortent de l'ombre, bravant ainsi les interdits qui leur sont imposés par nos hommes fanatiques, celles-ci sont tout simplement merveilleuses. (www.emarrakech.info, 2005-05-25)

53 Au singulier, en revanche, les emplois sont nettement plus contrastés selon le nombre du déterminant possessif. A la première pers. du pluriel, le SN notre homme traduit une forme d'empathie de la part du locuteur vis-à-vis d'un référent préalablement introduit dans le discours (33) :

(33) Devant un auditoire envoûté, notre homme venait de tirer les leçons définitives de la catastrophe de Fukushima en expliquant que, si les pompes avaient été situées quelques mètres plus haut, la centrale fonctionnerait toujours. ( www.monde-diplomatique.fr, 2011-10-07) 
54 A la $1^{\text {ère }}$ pers. du singulier (absent du notre corpus), mon homme suscite, une toute autre interprétation et indique une relation intime. Cette forme est censée émaner d'un parler populaire d'après le TLFi (34-35) :

(34) Elle attendit respectueusement que son homme voulût bien s'expliquer davantage (Quéffélec, Recteur, 1944 : 70). (TLFi)

(35) [...] je l'adore mon nom Lucie. Et quand mon homme Vincent me le chuchote à l'oreille... (Forlani, Gouttière, 1989)

\subsubsection{Détermination 0} personne (40):

(39) Qu'il sache qu'elle vit sa vie indépendante dans la ville la nuit, vaque à ses occupations syndicales, en femme militante libre de son temps, qu'elle va au cinéma si ça lui chante, pas du tout inféodée, sans concession ni joug qui tienne. (Garat, Pense à demain, 2010)

(40) Certes, je vous accorde que notre grosse duchesse est extraordinairement débraillée ; mais elle l'est en personne qui est à la tête des débraillés et qui, par conséquent, le doit être plus que les autres ; pour le surplus... (Chandernagor, L'Allée du Roi, 1981)

59 Le second type de constructions est marqué par des appositions. Celles-ci constituent presque un quart des fonctions grammaticales occupées par homme au singulier dans notre corpus (41-42). Nous aurons l'occasion d'y revenir.

(41) Mais Zakaria Boualem, homme plein de ressources, a une autre solution. ( www.telquel-online.com, 2011-10-18)

(42) Azouz Begag, qui s'est rangé dès le départ auprès du centriste François Bayrou, troisième homme de cette présidentielle selon les sondages, a été obligé de démissionner $\mathrm{du}$ gouvernement pour reprendre sa liberté de parole. ( www.aujourdhui.ma, 2007-04-10)

60 Au pluriel, le déterminant 0 apparait, dans la moitié des cas, dans des séquences énumératives et/ou dans des constructions manifestant un contraste " homme-femme " (43-44). Ce type de SN apparaît aussi en fonction de complément du nom, marquant la finalité du référent désigné par le nom-tête (mode/sport/rayon (pour) homme) (45) : 
(43) L'hôtellerie accueille jeunes et adultes, hommes et femmes, groupes et personnes individuelles pour un séjour de retraite et de recueillement. ( www.orval.be, 0002-11-30)

(44) France Retraite, filiale du groupe Caisse des Dépôts spécialisée dans les bilans prévisionnels de retraite, souligne l'inégalité de traitement entre hommes et femmes engendrée par le système actuel des retraites. (www.latribune.fr, 2007-02-28 )

(45) A céder salon de coiffure hommes, à Chalon-sur-Saône. (www.cm-saone-etloire.fr.txt, 2007-10-22)

\subsubsection{L'indéfini ${ }^{12}$}

61 Le déterminant indéfini prévaut au singulier, avec un pourcentage de $50 \%$, alors que les occurrences du pluriel en comptent 5 fois moins (9,5\%). Les séquences un homme / des hommes servent à introduire un référent "brand new» comme en (46-47-48) mais également, dans $13 \%$ des cas, à exprimer le SN en fonction d'attribut (49) :

(46) De son côté, Roxy s'éloigne et finit par rencontrer un homme mystérieux. ( Résumé des épisodes d'EastEnders, 2007-10-22)

(47) Quelques heures auparavant, à Mardan, petite ville du nord-ouest située non loin des fiefs talibans, un homme avait fait exploser sa bombe sur le trottoir, tuant un passant et en blessant quatre autres, selon la police locale. (www.latribuneonline.com, 2010-09-07)

(48) Dès la plus lointaine Antiquité, des hommes venaient ici pour prier, adorer, servir Dieu et leurs frères. (www.saint-loup.ch, 0002-11-30)

(49) Boulanger est un homme calme et rangé, d'allure propre, sans aucun tatouage et en bonne forme physique. (www2.canoe.com, 2009-04-17)

62 Les SN indéfinis sont également exploités dans des emplois qui mériteraient d'être approfondis, où le référent, pourtant déjà connu du locuteur et de l'interprète, se trouve littéralement re-présenté/re-saisi comme en première mention (50), là où un $\mathrm{SN}$ démonstratif ferait parfaitement l'affaire (51) :

(50) La prestation de serment, ce jeudi, du Dr Goodluck Jonathan comme président et chef suprême des Forces armées du Nigeria marque l'apogée d'un homme à l'ascension fulgurante. (www.grioo.com, 2010-05-07)

(51) La prestation de serment, ce jeudi, du Dr Goodluck Jonathan comme président et chef suprême des Forces armées du Nigéria marque le sommet d'une vie politique commencée en 1998 (Témoignages.re)

\subsubsection{Les quantifieurs}

Les quantifieurs sont l'apanage des SN pluriel. Ils arrivent en seconde position après le défini, avec un pourcentage de $20 \%$ des cas. Ils sont, dans $15 \%$ des cas assortis de déterminant défini (54) ou démonstratif (55) et sont instanciés principalement dans des faits divers ou comptes-rendus sportifs :

(52) En Californie, trois hommes accusés d'avoir allumé un feu de broussaille qui a détruit une cinquantaine de résidences de Malibu, en novembre dernier, ont plaidé non coupables. (lcn.canoe.com, 2007-12-22)

(53) Deux hommes ont été grièvement blessés dimanche lors d'un lâcher de taureaux à Pampelune. (www.europe1.fr, 2009-07-13)

(54) Davydenko vendange quatres (sic) coups droit de suite et les deux hommes reviennent à égalité. (www.20minutes.fr, 2007-06-05)

(55) Au-delà des colères provoquées par mon départ, ces deux hommes savent agir en stratèges. (www.eurosport.fr, 2007-04-05) 


\subsubsection{Le défini et le démonstratif}

\section{(56-58) :}

(56) Une phalange coupée Le traitement sédatif s'est toutefois avéré insuffisant chez cet homme puisque dans la nuit il a, à nouveau, agressé un autre infirmier qui venait le voir. (rss.feedsportal.com, 2009-11-30)

(57) Bonsoir Sandra, juste un petit retour positif pour vous confirmer la rencontre sentimentale que vous aviez vu (sic), elle a eu lieu effectivement et les descriptions que vous m'aviez faites de cet homme correspondent exactement. (www.crystalenergies.fr, 0002-11-30)

(58) L'homme à la crinière blanche et à la voix grave semble faire fi de la polémique née sitôt l'hypothèse Val évoquée dans les médias, décrivant l'ancien humoriste comme un homme cassant voire sectaire. (rss.feedsportal.com, 2009-06-17)

67 où le pluriel neutralise la distinction masculin/féminin (que nous appelons donc « neutre ») des cas où il renvoie à des référents masculins.

Comme le montre le tableau (3), les emplois neutres $\left(\mathrm{SN}_{\mathrm{N}}\right)$ où homme renvoie à l'espèce humaine (59-60) sont minoritaires (15\%) par rapport à ceux où le trait /+mâle/ est marqué (40\%).

Les emplois pluriels neutres sont majoritairement non modifiés (dans deux tiers des cas). Ils sont, par ailleurs, non spécifiques au sens où ils font référence à l'ensemble de l'humanité. Aucun n'est anaphorique :

(59) Cette méfiance à l'égard des hommes pousse le système à concevoir des normes rigides, en mesure de réduire, voire d'anéantir, les marges d'appréciation laissées à ses destinataires. (www.reds.msh-paris.fr.txt, 2007-10-22)

(60) Mais, aujourd'hui, les hommes s'aperçoivent que, même abondante, l'eau doit être protégée. (www.tsr.ch, 2011-10-19)

70 Concernant les cas de SN définis dotés du trait /+mâle/ ils sont, sous le rapport de la spécificité/non spécificité, dominés, quoique de peu, par l'emploi spécifique. Comme le montre le tableau (3), la différence entre les deux n'est que de $2 \%$. Les emplois spécifiques sont, qui plus est, caractérisés par une construction assez particulière qui prévaut sur le reste des emplois, à savoir :

Les hommes de Npr (18\%) (soit 85\% des cas de SN spécifiques) :

(61) Les hommes de Pierre Berbizier, qui viennent de perdre deux rencontres d'affilée, doivent absolument réagir et dominer à Colombes un concurrent direct pour les phases finales. (fr.sports.yahoo.com, 2008-05-29)

(62) Alors qu'ils avaient parfaitement débuté la rencontre avec un pressing haut et des intentions offensives clairement affichés, les hommes de Puel ont dès lors revu leurs plans en se recentrant les tâches défensives. (www.maxifoot.fr, 2006-11-02) 
Il est à noter que 27 d'entre eux sont anaphoriques (soit 13.5\% du total des occurrences et 34.5\% des occurrences d'hommes en emploi spécifique).

Pour ce qui concerne les emplois des SN définis non spécifiques où homme est sexué, la moitié sont utilisés sans modifieurs. Un tiers sert à marquer un contraste homme-femme (63-64) :

(63) L'élévation du risque de transmission concerne donc autant les hommes que les femmes. (www.rewmi.com, 2011-10-15)

(64) Nous chantons des chansons qui racontent les luttes des hommes et des femmes contre l'oppression et l'injustice, des chansons qui expriment notre colère, nos rêves, nos idéaux ou nos espoirs. (www.maretmanu.org, 0002-11-30)

\begin{tabular}{|c|c|c|c|}
\hline & \multicolumn{2}{|c|}{$\begin{array}{l}\text { SN défini pluriel les } \\
\text { hommes }\end{array}$} & \multirow[t]{2}{*}{ Total } \\
\hline & spécifique & Non spécifique & \\
\hline Neutre & \multicolumn{2}{|l|}{$15 \%$} & $15 \%$ \\
\hline /+ mâle/ & $21 \%$ & $19 \%$ & $40 \%$ \\
\hline TOTAL & $21 \%$ & $34 \%$ & $55 \%$ \\
\hline
\end{tabular}

Tableau 3. Usage du défini pluriel d'hommes ${ }^{13}$

\subsubsection{Les fonctions grammaticales}

Au plan des fonctions grammaticales occupées par les SN, le tableau 4 montre, dans l'ensemble, assez peu de différences entre les fonctions (objet, circonstant, CDN et autres). Seules deux d'entre elles se différencient assez nettement :

- celle de sujet, qui est occupée par les SN pluriel dans plus de la moitié des cas (51.5\%) et dans un tiers des cas au singulier ( $35 \%$ très exactement)

- celle d'attribut et apposition, qui fait un cinquième des emplois du SNsg et qui est dix fois moins occupée en tant que telle par les SNpl.

\begin{tabular}{|l|l|l|l|l|l|l|}
\hline & Sujet/agent & objet & circonstant & $\begin{array}{l}\text { Attribut } \\
\text { apposition }\end{array}$ & Ct du N/Ad & autre \\
\hline homme & $35 \%$ & $11.5 \%$ & $6 \%$ & $23.5 \%$ & $21 \%$ & $3 \%$ \\
\hline hommes & $51.5 \%$ & $16 \%$ & $8 \%$ & $2.5 \%$ & $21 \%$ & $1 \%$ \\
\hline
\end{tabular}

Tableau 4. Répartition des fonctions grammaticales d'homme au sg/pl.

\subsubsection{La fonction sujet}

La fonction de sujet se répartit différemment au sein des $\mathrm{SN}$ pluriel. En effet, les $\mathrm{SNpl}_{\mathrm{N}}{ }^{14}$ et $\mathrm{SNpl}_{\mathrm{M}}$ non spécifiques totalisent $15 \%$ des occurrences du pluriel alors que les $\mathrm{SNpl}$ spécifiques s'élèvent à $34 \%$, soit un peu plus du double que les non spécifiques. Il faudrait procéder à une étude systématique des verbes dont ces différents SN sont sujets, mais, en tout état de cause, il ne s'agit pas majoritairement de verbes « d'action », entendus ici au 
sens large du terme, mais plutôt des verbes statifs ou des attributifs, comme l'illustrent (65-68) :

(65) Ce que les hommes acceptent de reconnaitre comme musical correspond désormais à une appropriation d'un matériau sonore étendu, à une intégration de phénomènes jusqu'alors considérés comme bruits. (Musique, 2007-10-22)

(66) Mais, aujourd'hui, les hommes s'aperçoivent que, même abondante, l'eau doit être protégée. (www.tsr.ch, 2011-10-19)

(67) Les hommes préfèrent porter leur sac en bandoulière. (www.lemonde.fr, 2007-12-10)

(68) Les hommes sont sacrément bon public. (www.hebdo.ch, 2010-01-04) spécifiques (69):

(69) On m'a dit qu'un homme devait retenir les larmes le plus longtemps possible, c'est ce que j'ai fais. (generationrose.blogspot.com, 2007-12-07)

\section{Quant aux SNsg spécifiques sujets, un bon quart le
de verbes attributifs ainsi que de verbes au passif:}

(70) Un employé de la scierie serait alors sorti du bureau pour constater qu'un homme inconscient gisait ensanglanté à l'intérieur de son véhicule. www.cyberpresse.ca, 2005-10-25)

(71) Cet homme est notamment propriétaire d'un sac contenant une kalachnikov, d'un chargeur et d'un gilet pare-balles, selon le parquet de Paris. ( www.francesoir.fr, 2010-05-23)

(72) L'autre homme inculpé a été relaxé. (www.letelegramme.com, 2009-08-12)

(73) Un homme suspecté d'être l'agresseur a été interpellé. (www.francesoir.fr, 2010-05-28)

\subsubsection{La fonction d'attribut/apposition}

Cela étant, la fonction qui départage massivement le singulier et le pluriel est celle d'attribut ou d'apposition (que nous considérons ici, ainsi qu'il est admis généralement, comme une forme de prédication seconde).

Dans ces constructions, homme n'est employé que dans 3\% du total des cas sans modifieur. Ramené au total des cas d'attributs/appositions, cela correspond à $1 / 3$ des emplois qu'illustrent (74) et (75):

(74) Benjamin Garcia n'admet pas que les autorités municipales l'aient empêché l'an dernier de participer au concours parce qu'il est un homme. (www2.canoe.com, 2008-11-09)

(75) Des années plus tard, on retrouve Conan, maintenant devenu un homme, jouant les Robins des bois avec un comparse incarné par l'acteur noir imposé par la censure américaine. (nemedie.free.fr, 0002-11-30)

Les deux tiers restants (qui correspondent à $8 \%$ du total des occurrences) sont des $\mathrm{SN}$ modifiés, parfois par des compléments adnominaux qui servent à ranger le référent dans des sous-classes (76-77) ou par des adjectifs dénotant des propriétés du référent (78-79) :

(76) Un grand protecteur du monde littéraire Dorset, lui-même homme de lettres, fut un généreux protecteur du monde littéraire. (fr.wikipedia.org, 2009-06-14)

(77) Schoelcher est un homme d'Etat, un homme politique

(78) Vous dites souvent que vous n'êtes pas un homme riche, maintenant vous l'êtes enfin! (www.lematin.ch, 2009-03-04)

(79) Je trouve que Stéphane à l'air d'un homme drôle, évidemment, enjoué et surtout sociable. (www.voir.ca, 2006-10-12) 
81 Pour étayer ces données et nous assurer que ce faible pourcentage n'est pas induit par la taille et la nature de notre corpus, une enquête complémentaire, menée dans Frantext, montre que les séquences est un homme sont peu nombreuses. Elles équivalent à 3949 occurrences $^{15}$, ce qui correspond à $1 \%$ à peine du total de mots de la base. Par ailleurs, les énoncés collectés ne servent jamais de phrases définitoires. En effet, les séquences-types que nous avons trouvées se répartissent en deux groupes.

Le premier groupe est constitué des énoncés, dont le SN1 est/renvoie à un référent spécifique, servant principalement à caractériser le référent par des éléments descriptifs (80-81) ou à identifier un référent non identifiable au départ (82) (cf. Mahlberg, 2005) :

\section{i) Rft Spécifique est un homme + expansions descriptives}

(80) Un paysan d'un hameau voisin sort de l'épicerie au moment où je vais rentrer dans la maison. Il vient vers moi. C'est un homme taciturne et renfermé (Picquet, Sans illustration, 2013)

(81) Je pénètre enfin dans la grande salle obscure, cherchant parmi les box occupés la silhouette attendue, la longue natte tressée. Surprise : c'est un homme qui me fait signe - de petite taille mais vif - pour que je me joigne à lui. Maître Henri Leclerc - ce ne peut être que lui - m'embrasse sans hésiter et nous nous asseyons. (Castel, Retour d'exil d'une femme recherchée, 2009)

\section{ii) Rft Spécifique est un homme}

(82)

Qu'est-ce que tu regardes? demanda-t-elle.

- Les dessins...

Baissa son menu et suivit le profil de son frère.

- À ton avis, c'est un homme ou une femme ? continua-t-il.

- De quoi ? Ce dos, là ? (Gavalda, La Consolante, 2008)

Dans le second groupe, le référent sujet de la prédication n'est pas spécifique et la prédication vise soit à le classer (83-84) et/ou à souligner le trait /+mâle/ (85) :

\section{iii) Phrase classifiante}

(83) il devient presque impossible quand il y a une flambée de situations intolérables, comme le refus des soins donnés aux femmes dans les hôpitaux quand le médecin est un homme. (Ozouf, Composition française : retour sur une enfance bretonne, 2009)

(84) Benjamin Garcia n'admet pas que les autorités municipales l'aient empêché l'an dernier de participer au concours parce qu'il est un homme. (www2.canoe.com, 2008-11-09)

\section{iv) Enoncés pseudo-tautologiques du type un homme est un homme}

(85) Mais moi, je l'avais vu. A présent, je savais que le dirigeant de Fiat avait des problèmes de caleçon, comme tout le monde. Dans Les Justes - je l'ai vérifié par la suite - la terroriste Dora évoquait déjà un aléa de ce type lors d'un attentat. "Oh! Yanek, disait-elle, il faut que tu saches, il faut que tu sois prévenu ! Un homme est un homme (Osmont, Éléments incontrôlés, 2012)

\subsubsection{Bilan}

Pour synthétiser le propos, quatre points ressortent de cette étude de corpus. Premièrement, homme est utilisé majoritairement comme représentant du sexe masculin. Deuxièmement, la distribution entre le singulier et le pluriel montre une nette complémentarité, notamment au plan de la détermination : le singulier étant déterminé préférentiellement au moyen des déterminants indéfini, 0 , démonstratif et défini, le 
pluriel l'étant plutôt par les quantifieurs et le défini. Au plan des fonctions grammaticales, le singulier est usité dans des fonctions comme les attributs /apposition qu'ignore le pluriel. Troisièmement, les emplois spécifiques dominent, ils englobent la totalité des occurrences au singulier et un peu plus de la moitié des pluriels. Enfin, un tiers des emplois du singulier sont anaphoriques, contre $14 \%$ des pluriels. En outre, cette étude va nous permettre de préciser la place du NH homme au sein des NG.

\section{Retour sur les noms généraux}

Hors étude de corpus, nous avons vu, plus haut, que homme vérifie trois des caractéristiques des noms généraux: la commutabilité avec des pronoms, la fonction cohésive et la dimension interpersonnelle. Notre étude de corpus permet de nuancer ces points.

\subsection{Homme est-il un nom général ? Nuances}

Le premier point est difficile à évaluer compte tenu de notre corpus et de l'étroitesse des fenêtres donnant accès au contexte.

91 La fonction cohésive est confirmée par le taux d'emplois anaphoriques, même si elle semble plus manifeste au singulier ( $30 \%$ d'anaphores) qu'au pluriel où l'on ne décompte que $14 \%$ d'anaphores. Ces pourcentages montrent que les anaphores par hyperonymes/ termes généraux ne sont pas aussi répandues qu'on pourrait le croire. Voir à cet égard l'observation de [Reichler-]Béguelin (1995) qui vaut pour les noms qu'elle appelle générique et que nous corroborons dans notre étude de $2015^{16}$ :

La routine discursive qui consiste à rappeler par un nom générique un objet-dediscours préalablement désigné par un nom spécifique, quoique non préconisée par

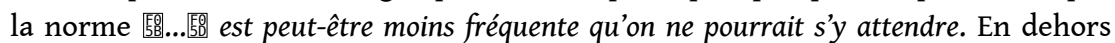
des taxinomies scientifiques, il y a d'ailleurs souvent un problème pour mettre en relief l'implication lexicale stricte entre les lexèmes concernés. ([Reichler]Béguelin, 1995 : 68 ; les italiques sont de nous)

La dimension interpersonnelle transparaît à travers deux emplois: les emplois du possessif que nous avons qualifié d'« empathique» (cf. supra, 3.2.2.1.) (cf. aussi 86-87); elle pourrait l'être aussi à travers la construction en homme illustrée plus haut et cidessous (88-91) :

(86) Entre-temps, notre homme aura fui tant et plus, de Shanghai à Pékin, et l'île d'Elbe. (www.liberation.fr, 2005-11-08)

(87) Devant un auditoire envoûté, notre homme venait de tirer les leçons définitives de la catastrophe de Fukushima en expliquant que, si les pompes avaient été situées quelques mètres plus haut, la centrale fonctionnerait toujours. ( www.monde-diplomatique.fr, 2011-10-07)

(89) Vivre, mourir en hommes. Et tous, ils moururent en hommes! (SaintExupéry, Citad., 1944, p. 554). Maman décida de le " traiter en homme » (Guéhenno, Jean-Jacques, 1948, p. 73)

(90) Tyrone Meehan, qui m'a expliqué que, pour pisser en homme, il fallait accepter de se montrer en homme (Chalandon, Mon traitre, 2007)

(91) Reconnais donc loyalement ton fiasco. Quelles conclusions en tirer ? Une et une seule : réagir en homme. Te ressaisir. Tu ne peux pas accepter de rester un jour de plus dans cet état. (Bénabou, Écrire sur Tamara, 2002) 
93 A ce point de vue, une piste de réflexion consisterait à s'interroger sur les différences dans les constructions attributives notamment entre (92a-92b) et (93a-93b-93c), sémantiquement très proches en apparence. Elles posent la question de la présence de homme dans le syntagme :

(92a) Alex Ferguson est un homme prudent. (www.varmatin.com, 2011-10-22)

(92b) Alex Ferguson est prudent

(93a) Pierrot est un homme malheureux

(93b) Pierrot est un malheureux

(93c) Pierrot est malheureux

On observera aussi que la tournure exclamative (94-96) - qui ne semble guère possible avec des termes synonymes d'homme (95) - semble inciter d'emblée à une appréciation positive :

(94) Quel homme!

(95) *Quelle personne ! *uels gens ! \#17 Quel individu !

(96) Cela vous surprendrait-il si je vous disais que mon pauvre mari entretenait une relation suivie avec Aymard de Montserrat, relation qui s'était malheureusement interrompue entre nos deux maisons depuis le XIII ${ }^{\text {e }}$ siècle à la suite d'un très regrettable incident? Ah cet Aymard, quel homme ! Et croyez-moi sa nièce est de la même trempe, un vrai garçon manqué ! (J. Lanzmann, La Horde d'or, 1994)

\subsection{Sens d'homme et dépendance cotextuelle}

Parmi les caractéristiques des noms généraux susceptibles de s'appliquer aux $\mathrm{NH}$, non prises en compte jusqu'à présent, restent : i) la dépendance cotextuelle du sémantisme de homme et ii) sa dimension « hyperonymique ».

Concernant le premier point, il est clair que l'interprétation d'homme varie selon les contextes, ce que prouverait la diversité des synonymes qui lui sont reconnus. En effet, pour en dresser un inventaire aussi exhaustif que possible, nous nous sommes appuyés sur trois ressources outre les dictionnaires d'usage: une base internet dénommée synonymo ${ }^{18}$, Dicosyn, le dictionnaire en ligne des synonymes établi par le CRISCO ${ }^{19}$ (cf. Figure 2), et le Wiktionnaire (entrée homme). Il résulte de cette enquête une liste autrement plus importante que ce qui est dit d'ordinaire, puisqu'elle totalise quelque 80 unités comprenant non seulement les termes relativement attendus d'humanité, personnage ou humain mais aussi d'autres, plus surprenants, comme gazier, ouvrier, ou moineau. A titre d'exemple, voici le début de la liste proposée par Dicosyn : 


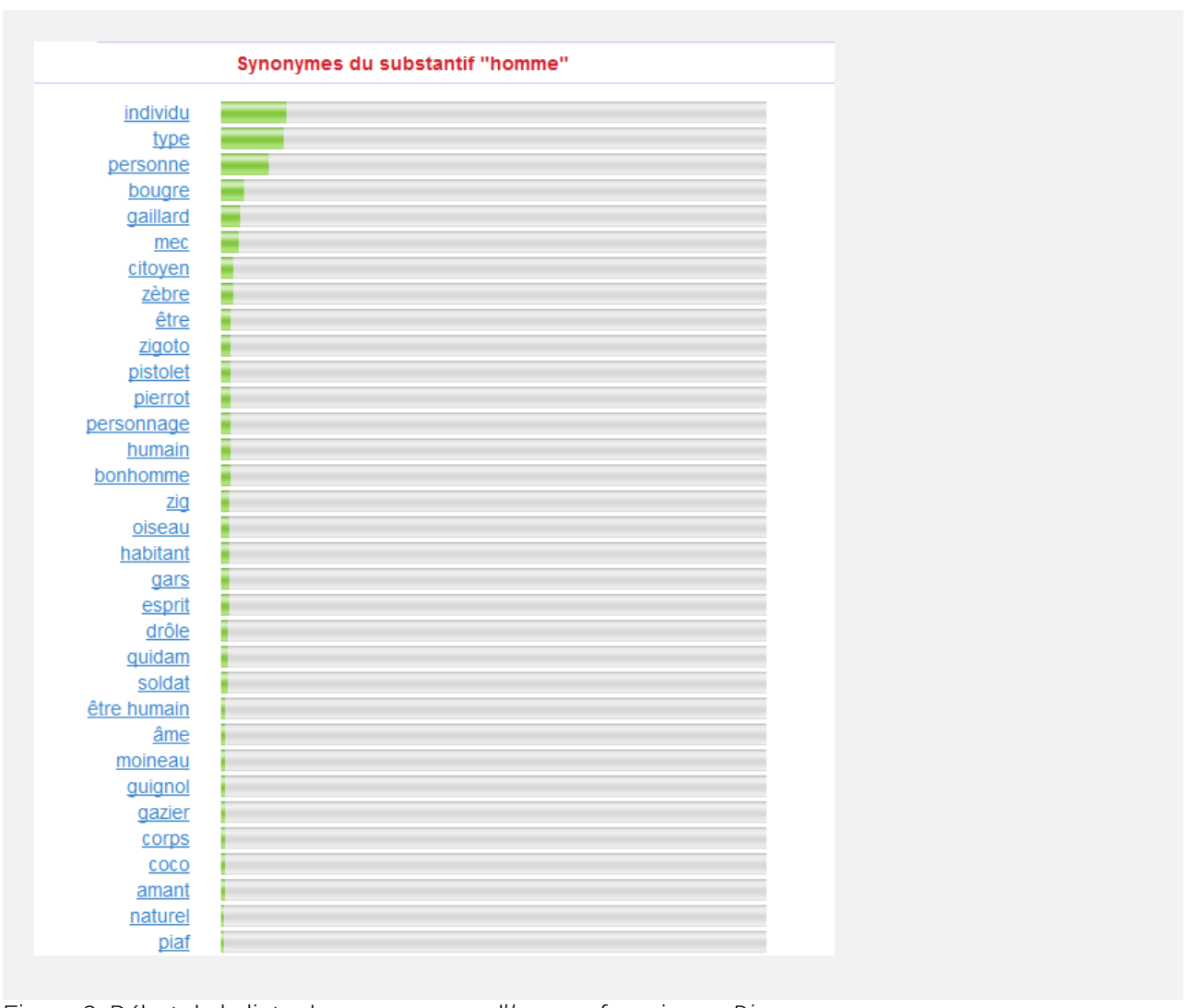

Figure 2. Début de la liste des synonymes d'homme fournie par Dicosyn.

Or, la commutation par les trois premiers termes qui font office des synonymes les plus fréquemment cités ne va, pour autant, pas de soi $^{20}$ :

(97) L'élévation du risque de transmission concerne donc autant les hommes que les femmes. (www.rewmi.com, 2011-10-15)

(98) *L'élévation du risque de transmission concerne donc autant les [individus/ types/personnes] que les femmes.

(99) Le principal suspect serait en effet un homme d'origine irakienne résidant à Luton et lié à Al-Qaïda. (www.parismatch.com, 2011-10-13)

(100) Le principal suspect serait en effet un(e) [individu/ ?type ${ }^{21} /$ personne] d'origine irakienne résidant à Luton et lié à Al-Qaïda.

Ceci expliquant en partie cela, la polysémie de ce nom a été maintes fois démontrée : l'article homme du TLFi tient sur une bonne dizaine de pages; la figure (3) empruntée à Baider \& Jacquey (2008) montre toute la complexité du terme : 


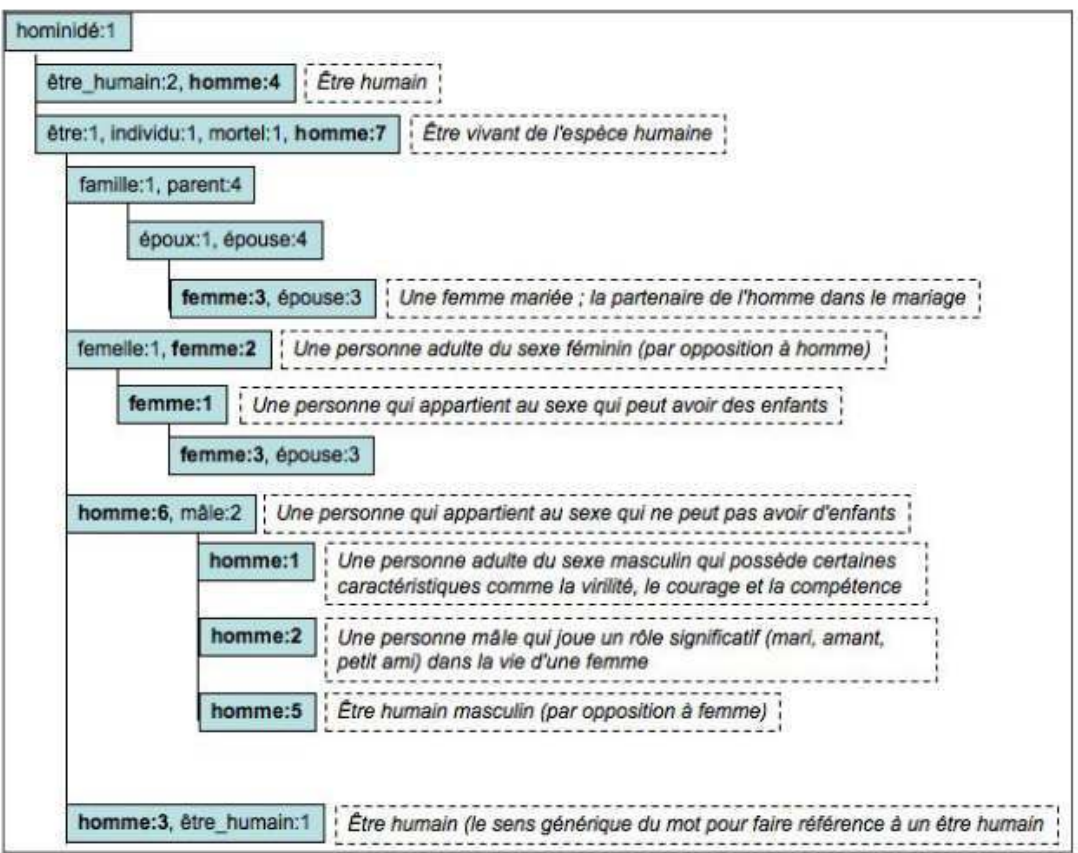

Fig 1: Représentation de homme et femme dans EuroWordNet

Figure 3. D’après Baider \& Jacquey (2008)

\subsection{Homme est-il un hyperonyme?}

Concernant la dimension hyperonymique, nous l'avons signalé (Schnedecker, 2015), le paradoxe de homme est qu'il renvoie à l'espèce humaine ce qui le situe au même niveau que « animal » et « végétal ». Voici les commentaires de Mortureux 1997 :

"Tantôt les trois mots animal, homme, végétal seront considérés comme cohyponymes de être vivant, tantôt homme fonctionnera comme un hyponyme d'animal dans la tripartition des règnes (subdivisions naturelles) minéral, végétal, animal ; c'est dans le cadre de cette tripartition que l'on dit l'homme est un animal doué de raison. [...]

Dans les exemples ci-dessus, homme a la valeur d'« être humain », représentant de l'espèce humaine ; mais si on le prend avec la valeur " mâle de l'espèce humaine », il est un hyponyme de mâle... Autrement dit, il peut être tantôt hyperonyme, tantôt hyponyme de mâle, en fonction de ses deux acceptions (humain (mâle ou femelle) ou mâle de l'espèce humaine). » (Mortureux 1997 : 84)

100 Autrement dit, et comme l'explique clairement Koch (2005: 18), la solution adoptée par le français et d'autres langues romanes est la suivante :

Un trait typologique particulièrement saillant du français concerne la taxinomie interne du domaine conceptuel fondamental ETRE HUMAIN $(=\mathrm{C} 1)$ qui se divise en ETRE HUMAIN MALE $=$ C11 et ETRE HUMAIN FEMININ $=\mathrm{C} 12$. La solution française consiste à désigner $\mathrm{C} 1$ aussi bien que $\mathrm{C} 11$ par le mot homme dont la 'polysémie verticale' (Gévaudan, 2003) recouvre deux niveaux taxinomiques à la fois. Il n'y a que $\mathrm{C} 12$ qui soit lexicalisé séparément, par femme. La plupart des langues romanes et l'anglais appartiennent à ce même type $\mathrm{C} 1-\mathrm{C} 11 / \mathrm{C} 12$ (p. ex. espagnol hombre/mujer ; it. uomo/donna ; angl. man/woman) inspiré parait-il par un effet de prototypicalité. La conception patriarcale qui fait de l'être humain mâle l'être humain 


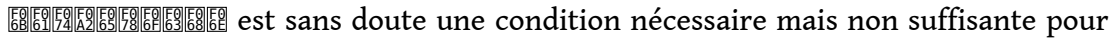
engendrer cette solution (...). (Koch, 2005, 18) déterminer si un $\mathrm{NH}$ comme homme participe de la catégorie des noms dits "généraux ». Pour ce faire nous avons repris les caractéristiques de ces noms de manière à voir si homme en était doté. Nous avons proposé des réponses en deux temps : certaines à partir de considérations " théoriques ", d'autres empiriques, à la lumière des usages manifestés par un corpus. En tout état de cause - de manière encore provisoire sans doute - il nous paraîtrait plus prudent de nous en tenir à l'étiquette de nom général pour ce qui est $\mathrm{d}$ 
'homme, ne serait-ce que pour éviter celle d'hyperonyme, qui ne s'est pas révélée totalement adéquate. Mais il faudrait poursuivre la réflexion en approfondissant certains des termes proposés par les spécialistes des NG comme ceux de vague et de sousspécification.

Cela étant dit, cette étude ouvre sur d'autres pistes de réflexion. Si le faible taux d'occurrences d'homme non spécifiques et neutres est sans nul doute un effet de notre corpus, il faudrait essayer de compléter cette étude en l'élargissant à des genres plus diversifiés de manière à capter l'ensemble des nombreuses valeurs d'un nom complexe comme celui-ci et de pouvoir véritablement les hiérarchiser. Dans un ordre d'idée similaire, il faudrait constituer un corpus qui serve à étudier l'ensemble des noms d'humains " généraux » - que ce soit sur le français ou dans une perspective contrastive (cf. l'étude de Roque Amaral \& Mihatsch W. (2016) et Mostrov \& Aleksandrova, dans ce volume) - de manière à ce que les résultats reposent sur des données comparables. Enfin, il faudrait bien sûr mener ce genre d'étude sur l'ensemble des synonymes d'homme, de manière à être réellement en mesure de fournir sinon une liste fermée des noms d'humains " généraux », du moins un noyau dur.

ROQUE AMARAL E. \& MIHATSCH W. (2016) Le nom français personne en comparaison avec le portugais brésilien pessoa et l'allemand Person : des noms en voie de pronominalisation? 5 ième Congrès Mondial de Linguistique française, juillet 2016. SHS Web of Conferences, Volume 27, 2016.

\section{BIBLIOGRAPHIE}

ADLER S. (2012) « Trois questions relatives aux noms généraux factuels attitudinaux », Scolia, 26, 11-37.

ARIEL M. (1990) Accessing Noun Phrase Antecedent, London, Routledge.

BAIDER F. \& JACQUEY E. (2008) « Modélisation sémantique, sens différentiel et genre », in J. Durand, B. Habert, B. Laks (éds), Congrès Mondial de Linguistique Française, https:// www.linguistiquefrancaise.org/articles/cmlf/pdf/2008/01/cmlf08240.pdf

BERRENDONNER A. (1983) «Connecteurs pragmatiques et anaphore », Cahiers de linguistique française , 5, 215-246.

BOLINGER D. (1977) Meaning and Form, London, Longman.

BOONE A. (1998) « Essai de typologie des phrases copulatives», M. Forsgren, K. Jonasson, H. Kronning (éds), Prédication, assertion, information, Actes du colloque d'Uppsala en linguistique française, Uppsala, Acta Universitatis Upsaliensis, 67-80.

CAPPEAU P. \& SCHNEDECKER C. (2014a) « Des gens bien différents à l'oral et à l'écrit », Verbum XXXVI, $1,55-74$.

CAPPEAU P. \& SCHNEDECKER C. (2014b) « Gens, personne(s), individu(s). Trois saisies de l'humain », in F. Neveu, P. Blumenthal, L. Hriba, A. Gerstenberg, J. Meinschaefer et S.Prévost (éds) $4^{\mathrm{e}}$ Congrès Mondial de Linguistique Française, (Berlin), SHS Web Conference, 3027-3040. [http:// 
www.shsconferences.org/articles/shsconf/abs/2014/05/shsconf_cmlf14_01274/

shsconf_cmlf14_01274.html]

CAPPEAU P. \& SCHNEDECKER C. (2015) « (Les/des) gens vs (les/des) personnes : évolution diachronique et comparaison diamésique. Des SN en voie de pronominalisation », in K. Jeppesen Kragh \& J.

Lindschouw (éds), Les variations diasystématiques et leurs interdépendances dans les langues romanes, Strasbourg, Travaux de linguistique romane, 449-463.

CAPPEAU P. \& SCHNEDECKER C. (2017) «L'oral des jeunes fait-il évoluer la langue ? », in F. Gadet (éd.) Les parles jeunes dans l'île de France multi-culturelle, Paris, Ophrys, 127-142.

CRUSE, ALAN (1986) Lexical Semantics, Oxford : Oxford U.P.

FLOWERDEW J. (2002) “A pedagogic Grammar of signaling Nouns in Discourse”, Revista Canaria de Estudios Ingleses, 4/4, 141-155.

FRANCIS G. (1986) Anaphoric Nouns, English Language Research, Department of English, Birmingham, University of Birmingham.

GROSS G. (1995) « A propos de la notion d'humain », in J. Labelle et C. Leclère (éds), LexiquesGrammaires comparés en français, Lingvisticae Investigationes Supplementa 7, Amsterdam, J. Benjamins, 71-80.

Gross, G. (2008) « Sur le statut syntaxique des substantifs humains », in D. Leeman (éd.) Des topoï à la théorie des stéréotypes en passant par la polyphonie et l'argumentation dans la langue, Paris, L'Harmattan, 27-41.

HALLIDAY M.A.K. \& HASAN R. (1976) Cohesion in English, London, Longman.

haspelmath, M. (1997) Indefinite Pronouns. Oxford. Clarendon Press.

HEINE, B. \& KYUNG-AN, S. (2011) “On the grammaticalization of personal pronouns”, Journal of Linguistics 47, 587-630.

JOHANSSON S. (2007) Seeing through Multilingual Corpora. On the use of corpora in contrastive studies, J. Benjamins.

косн Р. (2005) « Aspects cognitifs d'une typologie lexicale synchronique. Les hiérarchies conceptuelles en français et dans d'autres langues ", Langue française 145, 11-33.

LAKOFF, G. (1986) Women, Fire, and Dangerous Things, Chicago/London, Chicago Press University.

LARIVIÈRE (2001) « Typologie des noms communs de personne et féminisation linguistique ", Revue Québécoise de Linguistique 29/2,15-31.

LEGALLOIS D. (2008) «Sur quelques caractéristiques des noms sous-spécifiés », Scolia 23, 109-27.

LE PESANT D. (2002) « La détermination dans les anaphores fidèles et infidèles », Langages 145, 39-59.

LYONS J. (1978) Eléments de sémantique, Paris, Larousse.

MEDIN D, L., LYNCH, E. B., SOLOMON, K.O. (2000) “Are there Kinds of Concepts ?”, in Annual Review of Psychology 51, 121-147.

MAHLBERG M. (2005) English general nouns : a corpus theoretical approach, J. Benjamins.

MIHATSH W. (2015a) «La sémantique des noms généraux 'être humain' français et allemands », in W. Mihatsch \& C. Schnedecker C. (éds), Les noms d'humains : une catégorie à part ? Stuttgart, Steiner, (ZfSL-B) (Zeitschrift für französische Sprache und Literatur, Beiheft 40), 55-83. 
MIHATSCH W. (2015b) « La position taxinomique et les réseaux méronymiques des noms généraux 'êtres humains' français et allemands », in W. Mihatsch \& C. Schnedecker C. (éds), Les noms d'humains : une catégorie à part ? Stuttgart, Steiner, (ZfSL-B) (Zeitschrift für französische Sprache und Literatur, Beiheft 40), 85-113.

MORTUREUX M. F. (1997) La lexicologie entre langue et discours, Paris, Sedes.

PARTINGTON, A. (1998). Patterns and Meanings. Using Corpora for English Language Research and Teaching. Amsterdam: John Benjamins.

[REICHLER-]BÉGUELIN M.-J. (1989) « Anaphores, connecteurs, et processus inférentiels », in C. Rubattel (éd.), Modèles du discours. Recherches actuelles en Suisse romande, Berne, P. Lang, 303-336. [REICHLER-]BÉGUELIN (1995) « Alternatives et décisions lexicales dans l'emploi des expressions démonstratives », Pratiques 85, 53-87.

schmid H.-J. (2000) English Abstract Nouns as Conceptual Shells, Berlin- N.-Y. Mouton de Gruyter.

SCHNEDECKER C. (2015a) «Contraintes pesant sur les anaphores à nom général dans les chaînes de référence renvoyant à des entités humaines ", Travaux de Linguistique 70, 2015/1, 39-72.

SCHNEDECKER C. (2015b) « Les (noms d') humains sont-ils à part ? Des intérêts et perspectives linguistiques d'une sous-catégorie nominale encore marginale », in W. Mihatsch \& $\mathrm{C}$. Schnedecker C. (éds), Les noms d'humains : une catégorie à part ? Stuttgart, Steiner, (ZfSL-B) (Zeitschrift für französische Sprache und Literatur, Beiheft 40), 4-43.

SCHNEDECKER C. (2015c) «Un problème à la croisée des disciplines linguistiques : les noms d'humains comme interface entre morphologie, syntaxe et sémantique », in A. Rabatel et al. (éds) La sémantique et ses interfaces. Actes du colloque de l'Association des Sciences du Langages, 30/11/2013, Limoges, Lambert-Lucas, 111-141.

SCHNEDECKER C. (2017) «L'expression « indéfinie » de la personne par le SN dét+quidam : un cas de transcatégorialisation original ? » in B. Fagard, \& S. Prévost (éds) Le français en diachronie, Berne, P. Lang, 269-290.

WIERZBICKA, A. (1985) Lexicography and conceptual analysis. USA. Karoma Publishers.

\section{NOTES}

1. Cf. l'introduction à ce volume.

2. Sur ce point, Schnedecker (2015).

3. Voir la liste indiquée dans l'introduction à ce volume (1.1.).

4. Cf. Cappeau \& Schnedecker (soum.).

5. "Shell nouns serve the textual function of linking these nominal concepts with clauses or other pieces of text which contain the actual details of information, thereby instructing the hearer to interpret different sections of a text together". (Schmid, $2000: 14$ ).

6. Au niveau phonologique, ces emplois sont dépourvus en anglais d'accent tonique.

7. Ce qui fait que leur contenu n'est pas totalement stable: “ (...) the concepts created by shell nouns are also very variable. They are of temporary nature because their content change with the situational and linguistic context in which they are used. So the concept created by shell nouns consist of a stable symbolic and a variable indexical part". (Schmid, $2000: 18$ ).

8. Voir aussi le point de vue de Legallois (2008) sur les noms généraux (qu'il appelle noms sousspécifiés).

9. Schmid signale d'autres patrons: $N$-that, $N$-to, $N$-wh, $N$-be-that, $N$-be-to, $N$-be-wh (p. 22). 
10. http://corpora.informatik.uni-leipzig.de/\#! French mixed corpus based on material from 2012. Sentences: 74,823,426/ Types: 7,873,935/-Tokens: $1,468,766,604$.

11. Nous n'avons pas comptabilisé ici le $1 \%$ d'exclamatifs.

12. Nous n'avons pas trouvé dans notre corpus de un exprimant une quantification.

13. Les pourcentages sont calculés sur l'ensemble des occurrences d'hommes.

14. Les indices $\mathrm{N}$ et $\mathrm{M}$ indiquent respectivement «neutre » et « mâle ».

15. Rappelons que Frantext compte 285923119 occurrences, ce qui fait à peine $1 \%$ du total.

16. Et que nous essayons d'expliquer par un certain nombre de contraintes pesant sur l'emploi de ce type de $\mathrm{N}$ en anaphore.

17. \# signifie que l'énoncé est acceptable mais avec une interprétation différente de celle de (94).

18. http://www.synonymo.fr/

19. http://www.cnrtl.fr/synonymie/

20. Nous renvoyons au travail de W. Mihatsch (2015), d'une part et au nôtre (cf. Cappeau \& Schnedecker, 2014b, 2015) qui expliquent ces impossibilités par le sémantisme propre des $\mathrm{N}$ de personne et individu.

21. Type dépend comme le fait observer un relecteur, du niveau de langue, mais semble exclu de genres de la presse.

22. Terminologie de Boone $(1998 ; 76)$.

23. Dans les ouvrages traitant de taxinomies, classifications nominales, etc., les exemples éludent soigneusement l'humain.

\section{RÉSUMÉS}

Après avoir rappelé les caractéristiques des noms généraux tels qu'ils sont définis généralement dans la littérature et au rang desquels figure le nom homme, montre, sur la base d'une étude de corpus, à quel point l'usage est nuancé. En effet, homme est préférentiellement utilisé en étant doté du trait /+mâle/ et que les emplois génériques du nom, susceptibles de renvoyer à l'espèce humaine, sont minoritaires. A partir de là, elle opère un retour sur les critères dégagés pour définir les noms généraux et détermine la place que peut y occuper - sous certaines conditions homme.

After having pointed out the characteristics of the general nouns such as they are generally defined in the literature and with the row of which figure the noun homme, watch, on the basis of study of corpus, at which point the use is moderate. Indeed, homme is preferentially used with the feature/+mâle/and the generic uses of the noun are minority. From there, it operates a return on the criteria released to define the general nouns and determines the place which homme can occupy there - under certain conditions -.

\section{INDEX}

Mots-clés : sémantique nominale, noms généraux d'humains 
AUTEUR

CATHERINE SCHNEDECKER

Université de Strasbourg, LiLPa/Fonctionnements Discursifs \& Traduction 